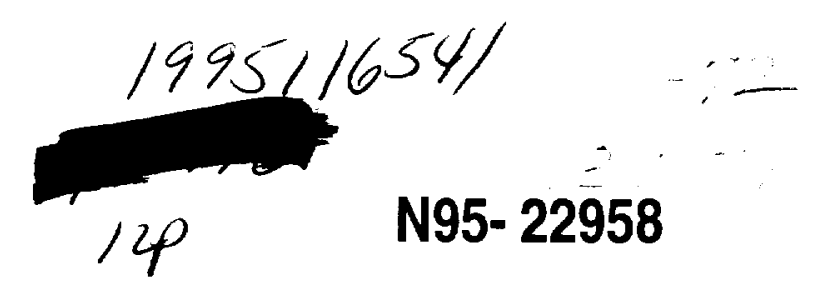

\title{
ALGEBRAIC THEORY OF MOLECULES
}

\author{
F. Iachello \\ Center for Theoretical Physics, Sloane Physics Laboratory, \\ Yale University, New Haven, CT 06520-8120
}

\begin{abstract}
An algebraic formulation of quantum mechanics is presented. In this formulation, operators of interest are expanded onto elements of an algebra, $\mathcal{G}$. For bound state problems in $\nu$ dimensions the algebra $\mathcal{G}$ is taken to be $U(\nu+1)$. Applications to the structure of molecules are presented.
\end{abstract}

\section{Introduction}

The development of new experimental techniques is giving rise to a wealth of information on complex systems. This information needs to be understood in terms of theoretical models which can, on one side, describe the observations and, on the other side, make predictions for other experiments. In view of the accuracy of the experiments, one needs new and more accurate mathematical models. For quantum mechanical problems, a natural framework is provided by the Schrödinger equation. In complex systems, the direct solution of the multiparticle Schrödinger equation becomes rather difficult and one many seek alternative methods. In this note, an alternative method, called algebraic theory, will be introduced and discussed briefly. The method will then be applied to the study of the structure of molecules. Problems of current interest in this field are, among others, the substitution of atoms in a large molecule leading to a lowering of symmetry, the polymerization process in which dimers, trimers, ... are formed from the original molecule, the study of new molecules, such as the cage fullerene molecules, $C_{60} ; \ldots$, and, particularly important, the study of molecules at high excitation energy. An example of the latter will be presented.

\section{Algebraic Theory}

The logic scheme of algebraic theory is as follows:

Quantum mechanical problem

$\Downarrow$

Algebraic Structure 

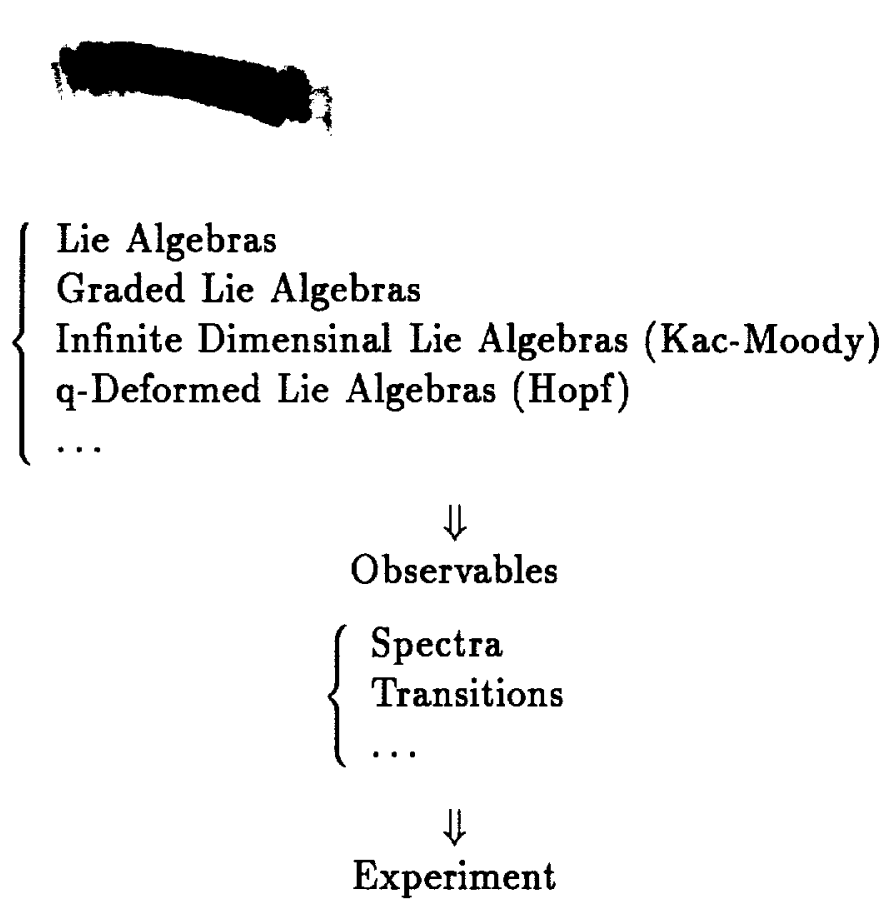

In the first step, all quantum mechanical operators of interest are mapped onto the elements of an algebra, $\mathcal{G}$. For example, the Hamiltonian operator is written as

$$
H=E_{o}+\sum_{\alpha} \epsilon_{\alpha} G_{\alpha}+\sum_{\alpha \beta} u_{\alpha \beta} G_{\alpha} G_{\beta}+\ldots, \quad G_{\alpha} \epsilon \mathcal{G}
$$

The algebra $\mathcal{G}$ is called the spectrum generating algebra (SGA) and $\mathrm{H}$ is in the enveloping algebra of $\mathcal{G}$.

In some cases, it may happen that the Hamiltonian $\mathrm{H}$ contains only certain elements of $\mathcal{G}$, the invariant Casimir operators of $\mathcal{G}$ and of one of its subalgebra chains, $\mathcal{G} \supset \mathcal{G}^{\prime} \supset \mathcal{G}^{\prime \prime} \supset \ldots$,

$$
H=f\left(C_{i}\right)
$$

This case, called a dynamic symmetry, plays a special role in algebraic theory, since then the eigenvalues of $\mathrm{H}$ can be obtained in closed analytic form in terms of the quantum numbers characterizing the representations of $\mathcal{G} \supset \mathcal{G}^{\prime} \supset \ldots$

Dynamic symmetries and spectrum generating algebras have been used in various contexts for more than 30 years [1]. As a result of the systematic investigation and use in the context of nuclear and molecular physics, initiated with the introduction of the interacting boson model [2] in 1974 and of the vibron model [3] in 1981, it has become clear that all quantum mechanical problems in $\nu$ space dimensions can be mapped onto the algebra $U(\nu+1)$ and all its states assigned to the totally symmetric representation $[\mathrm{N}]$ of $U(\nu+1)[4]$. Examples of this mapping are given in the following section.

\section{One dimensional problems}

To begin with, consider the single case of one space dimension, $\nu=1$. A trivial application of algebraic theory is provided here by the harmonic oscillator. The Schrödinger (differential) equation 


$$
H=\frac{1}{2}\left(-\frac{d^{2}}{d x^{2}}+x^{2}\right), H \psi_{n}=E \psi_{m}
$$

with eigenvalues $E_{n}=\left(n+\frac{1}{2}\right)$, and eigenfunctions

$$
u_{n}(x)=\left[\pi^{\frac{1}{2}} 2^{n} n !\right]^{-\frac{1}{2}}\left(x-\frac{d}{d x}\right)^{n} e^{-\frac{1}{2} x^{2}}
$$

(the Hermite polynomials), can be mapped into

$$
H=\left(a^{\dagger} a+\frac{1}{2}\right),
$$

with the same eigenvalues $E_{m}=\left(n+\frac{1}{2}\right)$ and eigenstates

$$
\left|n>=(n !)^{-\frac{1}{2}}\left(a^{\dagger}\right)^{n}\right| 0>\text {. }
$$

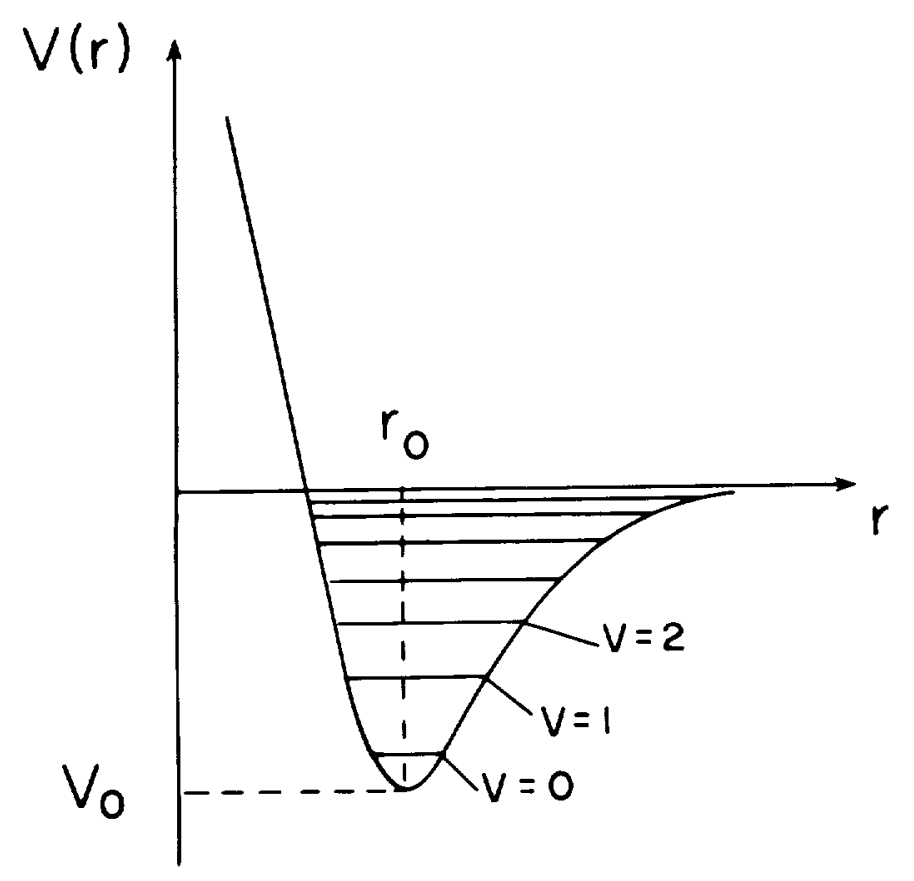

FIG. 1. The Morse potential V(r).

Here

$$
a=\frac{1}{\sqrt{2}}\left(x+\frac{d}{d x}\right), a^{\dagger}=\frac{1}{\sqrt{2}}\left(x-\frac{d}{d x}\right) \quad ; \quad\left[a, a^{\dagger}\right]=1
$$

The algebra is

$$
H(2)=a, a^{\dagger}, 1, a^{\dagger} a
$$


called the Heisenberg, quantum mechanical or oscillator algebras [5]. The mapping produces a great simplification both in the evaluation of the matrix elements of operators which are integrals in the differential formulation

$$
I_{n n^{\prime}}=\int_{-\infty}^{\infty} u_{n^{\prime}}(x) f\left(x, \frac{d}{d x}\right) u_{n}(x) d x
$$

and algebraic functions in the algebraic formulation

$$
I_{n n^{\prime}}=<n^{\prime}\left|f\left(a, a^{\dagger}\right)\right| n>
$$

A slightly more complicated problem is provided by the anharmonic Morse oscillator [6], Fig.1. The Schrödinger equation with

$$
H=-\frac{\hbar^{2}}{2 \mu} \frac{d^{2}}{d x^{2}}+V(x), V(x)=D[1-\exp (-\beta x)]^{2}
$$

with eigenfunctions

$$
\begin{aligned}
\psi_{v}(x) & =N_{s} z^{\eta-v} e^{-\frac{z}{2}+\frac{1}{2} x \beta} L_{v}^{2 \eta-2 v-1}(z) \\
z & =2 \eta e^{-\beta x}, \eta=\frac{1}{\hbar \beta} \sqrt{2 \mu D}, v=0,1, \ldots, \eta-\frac{1}{2}
\end{aligned}
$$

and eigenenergies

$$
E(v)=2 \hbar \beta \sqrt{\frac{D}{2 \mu}}\left(v+\frac{1}{2}\right)-\frac{1}{2} \frac{\hbar^{2} \beta^{2}}{\mu}\left(v+\frac{1}{2}\right)^{2}
$$

can be mapped onto the algebra $\mathcal{G}=U(2)$ with elements $F_{+}, F_{-}, F_{0}, N$ and corresponds to the dynamic symmetry $U(2) \supset 0(2)$ of this algebra. The Hamiltonian is

$$
H=A C \quad, \quad C=F_{0}^{2}-N^{2}
$$

with eigenenergies

$$
E(m)=A\left(m^{2}-N^{2}\right) ; m=N, N-2, \ldots, 1 \text { or } 0(N=\text { odd or even }) .
$$

The eigenvalues can be brought into the standard vibrational form introducing $v=(N-m) / 2$,

$$
E(v)=-4 A\left(N v-v^{2}\right) ; v=0,1, \ldots, \frac{N}{2} \text { or } \frac{N}{2}-\frac{1}{2}(N=\text { even or odd }) \text {. }
$$

With some small changes this is seen to correspond to Eq.(13). The eigenstates can be written as $|N, v\rangle$ and observables calculated as

$$
<N, v^{\prime}|\hat{T}(G)| N, v>
$$

where $\hat{T}(\mathrm{G})$ is the appropriate operator built from the elements of $\mathcal{G}$. By making use of the algebraic method, all results of the anharmonic Morse oscillator can be found easily. (Note that in 
the association of the Morse oscillator with $U(2) \supset O(2)$ only the positive branch of $O(2), m \geq 0$, has been used.)

As a third simple case, consider the anharmonic Pöshl-Teller oscillator

$$
V(x)=-\frac{D}{\cosh ^{2} \alpha x}
$$

This potential can also be associated with $U(2) \supset O(2)$ and Hamiltonian [7]

$$
H=A C
$$

with eigenvalaues

$$
E(v)=-4 A\left(N v-v^{2}\right)
$$

One can see from (16) and (20) that the Morse and Pöshl-Teller potentials have the same bound state spectrum (isospectral potentials). (This statement is not true in $\nu=2,3, \ldots$ dimensions.) The Morse and Pöshl-Teller potentials in $1 \mathrm{dim}$ belong to a class of potentials called exactly solvable since their eigenvalues can be written in explicit analytic form. All exactly solvable potentials in 1 dim have been classified.

The overall algebraic structure of 1 dim problems can be written as

$$
\begin{aligned}
& U(2) \quad \supset O(2) \approx U(1), \\
& \Downarrow c \\
& H(2) \supset O(2) \approx U(1) .
\end{aligned}
$$

In this equation, the arrow with a $c$ denotes a contraction of the algebra of $U(2)$. (In addition to the contraction $U(2) \vec{c} H(2)$, there is another one $U(2) \vec{c} E(2)$, where $E(2)$ is the Euclidean algebra not discussed here.)

\section{Multidimensional problems}

In more than one dimension, the connection between the Schrödinger equation and the corresponding algebraic equation is not so straightforward, with the only exception of the harmonic oscillator and Coulomb problem. It is here that algebraic methods are particularly useful, since by formulating directly the problem in an algebraic framework one can consiruct the spectrum and calculate observables without reference to a specific form of the potential. The algebraic structure of three dimensional problems can be written as $[4],[8]$

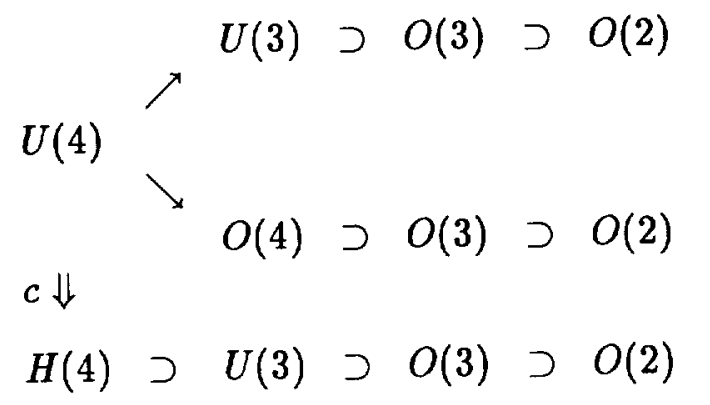


The chain (I) corresponds to Schrödinger problems with Pöschl-Teller-like potentials, the chain (II) corresponds to Morse-like potentials, while the chain (III) corresponds to the harmonic oscillator in $3 \mathrm{dim}$.

In general, in $\nu$ dim one has

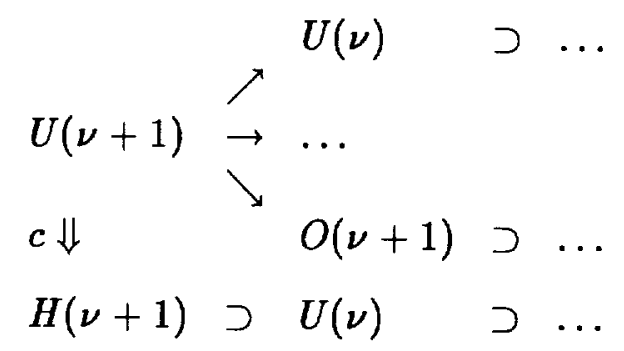

where now additional chains may appear in the reduction of $U(\nu+1)$. The five dimensional case, $\nu=5$, has been extensively investigated in the context of nuclear physics [9]

\section{Algebraic Theory of Large Molecules}

The algebraic approach of Sect. 2 can be used to study molecular structure. For reasons that will be mentioned in the subsequent section, it is convenient to separate large molecules from small molecules (large here means molecules with more than 4 atoms). In large molecules each degree of freedom, $\mathrm{x}, \mathrm{y}, \mathrm{z}$, is quantized with $U(2)$ and the total spectrum generating algebra is taken to be $\mathcal{G}=\sum_{i} \oplus U_{i}(2)[10]$. A calculation of spectral properties proceeds then as follows: In step 1, all atoms are numbered, $\mathcal{N}$; in step 2, three coordinates are assigned to each atom for a total of $3 \mathcal{N}$; in step 3, each coordinate is quantized with $U(2) \supset O(2)$, thus being treated as an anharmonic oscillator; in step 4, the oscillators are coupled with Hamiltonians

$$
H=\sum_{i=1}^{3 \mathcal{N}} h_{i}+\sum_{i \geq j=1}^{3 \mathcal{N}} w_{i j}
$$

The structure of the Hamiltonian (24), when written in terms of the elements of the algebra $\mathcal{G}$ is

$$
h_{i}=\epsilon_{o i}+A_{i}\left(\hat{F}_{o i}-N_{i}\right)^{2} \quad, w_{i j}=\lambda_{i j}\left(\hat{F}_{+i} \hat{F}_{-j}+\hat{F}_{-i} \hat{F}_{+j}\right)
$$

The $h_{i}$ terms are diagonal in the basis $U_{i}(2) \supset O_{i}(2)$ characterized by the quantum numbers $\left|N_{i}, v_{i}\right\rangle$ discussed in Sect. 3, while the $w_{i j}$ are given in Ref. [10],[11]. In the final step 5, the spurious species corresponding to overall rotations and vibrations, are identified and removed by diagonalizing the Harniltonian

$$
H^{\prime}=H+\lambda \mathcal{P},
$$

where $\mathcal{P}$ is a projection operator into the spurious species and $\lambda$ is taken to be a large number such that the spurious species are moved to a large energy. The removal of the spurious species 
leaves $3 \mathcal{N}-6$ non spurious vibrations. This procedure produces the vibrational spectrum of the molecule.

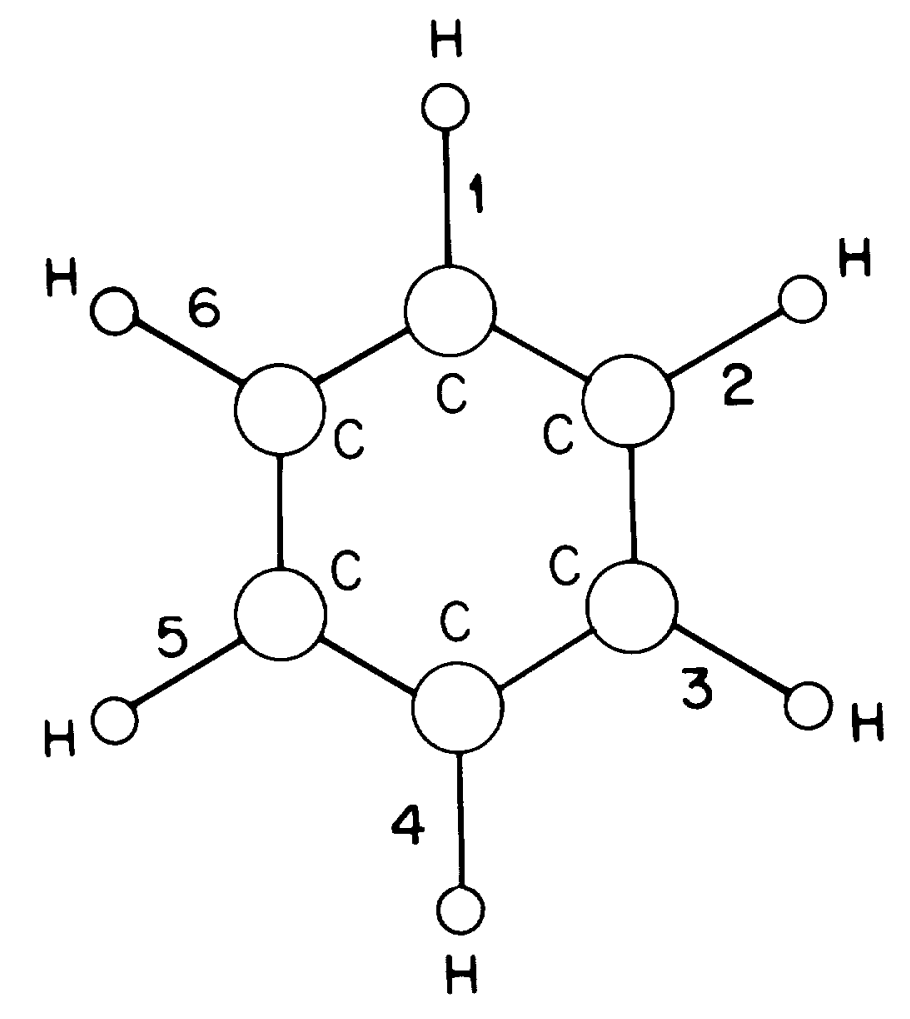

FIG. 2. Schematic representation of benzene $\left(C_{6} H_{6}\right)$.

In a similar way one can compute intensities of transitions. There are two types of transitions of importance in molecules, infrared (IR) and Raman (R) transitions. For infrared transitions, the appropriate operator is a vector. Each component $x, y, z$ of this vector ls written in terms of elements of $\mathcal{G}$, i.e.

$$
\hat{T}_{x}=\sum_{i=1}^{3 \mathcal{N}} \alpha_{i, x} \hat{t}_{i} \quad, \quad \hat{t}_{i}=e^{-\beta_{i}\left(\hat{F}_{+i}+\hat{F}_{-i}\right)} \quad, \quad \ldots
$$

The matrix elements of the operator (27) (and $\hat{T}_{y}, \hat{T}_{z}$ ) are then evaluated algebraically. For Raman transitions, the appropriate operator is a symmetric quadrupole tensor. The six components of this tensor, $x^{\mathfrak{Q}}, y^{2}, z^{2}, x y, x z, y z$, are also written in terms of $\mathcal{G}$ and their matrix elements evaluated algebraically.

As an example of vibrational analysis of large molecules, consider the case of benzene, $\mathrm{C}_{6} \mathrm{H}_{6}$. The benzene molecule has the geometric structure shown in Fig. 2. A problem which arises in large molecules is that of the discrete symmetry of the molecule. In the case of benzene, the 
appropriate symmetry is $\mathcal{D}_{6 h}$. The discrete symmetries of molecules can be simply implemented in the algebraic framework. For example, consider the six stretching vibrations of the hydrogen atoms in benzene. All hydrogen atoms are equivalent. The Hamiltonian (24) which describes those vibrations

$$
H=\sum_{i=1}^{6} A_{i} C_{i}+\sum_{i \leq j=1}^{6} \lambda_{i j} M_{i j},
$$

where $C_{i}$ and $M_{i j}$ are a short-hand notation for the terms in (25), must be such that one cannot distinguish the equivalent atoms. Thus, all $A_{i}$ 's must be equal, $A_{i}=\mathrm{A}$. In the interaction term, there are three contributions, first, second and third neighbor interactions. These too must be equal, $\lambda_{i j}^{(I)}=\lambda^{(I)}, \lambda_{i j}^{(I I)}=\lambda^{(I I)}$ and $\lambda_{i j}^{(I I I)}=\lambda^{(I I I)}$. The Hamiltonian $\mathrm{H}$ thus becomes

$$
H=A C+\lambda^{(I)} S^{(I)}+\lambda^{(I I)} S^{(I I)}+\lambda^{(I I I)} S^{(I I)},
$$

and is characterized by a smaller set of parameters. The operators $C, S^{(I)}, S^{(I I)}, S^{(I I I)}$ are appropriate linear combinations of the $C_{i}$ 's and $M_{i j}$ 's. A corollary of the algebraic method is that certain linear combinations of the operators $M_{i j}$ are symmetry adapter operators of $\mathcal{D}_{6 h}$ and the irreducible representations of $\mathcal{D}_{6 h}$ are obtained automatically by diagonalizing them [10],[12].

Using the algebraic method discussed above it has been possible to study the complete spectroscopy of benzene [13]. This molecule has 12 atoms and thus 36 degrees of freedom, 6 of which are spurious. The 30 non spurious species are shown in Table I.

TABLE I: Coordinates and symmetry species of benzene.

\begin{tabular}{|l|c|c|c|}
\hline Coordinates & Number & Species & Spurious \\
\hline CH stretch & 6 & $E_{2 g}+B_{1 u}+E_{1 u}+A_{1 g}$ & I \\
CC stretch & 6 & $A_{1 g}+B_{2 u}+E_{1 u}+E_{2 g}$ & \\
CH in plane bend & 6 & $E_{1 u}+B_{2 u}+E_{2 g}+A_{2 g}$ & \\
CH out of plane bend & 6 & $A_{2 u}+B_{2 g}+E_{1 g}+E_{2 u}$ & \\
CC in plane bend & $3+3$ & $B_{1 u}+E_{2 g}$ & $A_{1 g}+E_{1 u}$ \\
CC out of plane bend & $3+3$ & $B_{2 g}+E_{2 g}$ & $A_{1 u}+E_{1 g}$ \\
& & & \\
\hline
\end{tabular}

The calculation describes the observed vibrational states not only in the low excitation energy region, fundamental vibrations, but also in the high excitation energy region, overtones. An example is shown in Fig. 3. This region cannot be described in the harmonic approximation and thus the use of algebraic methods based on the anharmonic Morse or Pöschl-Teller oscillators are 
crucial for an accurate description of the observed spectra.
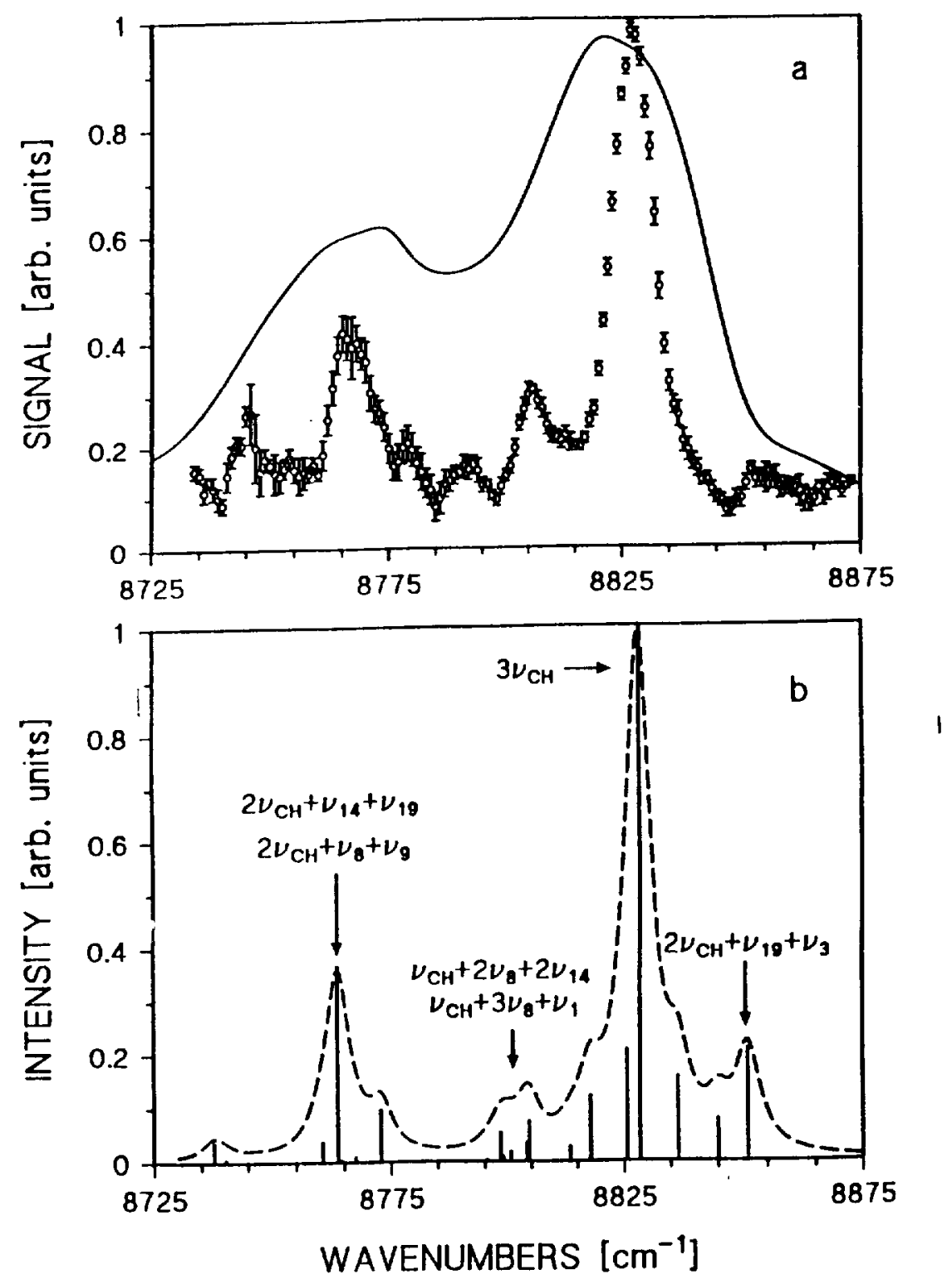

FIG. 3. (a) Opto-thermal spectrum in the region of the $\Delta v=3$ overtone of the stretching $\mathrm{CH}$ mode of benzene. The full-line is a low-resolution experiment. (b) The spectrum calculated by means of algebraic theory. From Ref.[14]. 


\section{Algebraic Theory of Small Molecules}

For molecules with a number of atoms less or equal to four, it is possible to quantize each vector degree of freedom, $\vec{r} \equiv(\mathrm{x}, \mathrm{y}, \mathrm{z})$, directly in terms of $U(4)$. When quantized in this way both rotations and vibrations are simultaneously included.

(b)

(a)

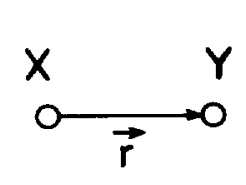

(c)

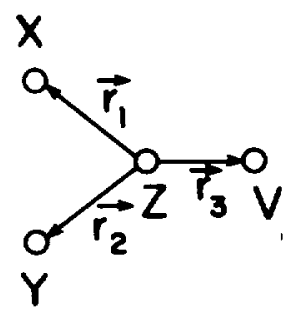

FIG. 4. Bond variables for small molecules.

It is also convenient to treat as vector variables the bond degrees of freedom, Fig.4, rather than the coordinates of the individual atoms, thus avoiding the problem of spurious states. The quantization scheme is thus here $\mathcal{G}=\sum_{i} \oplus U_{i}(4)$. This scheme has been extensively used to treat diatomic molecules with $U(4)$, triatomic molecules with $U(4) \oplus U(4)[15]$ and four atomic molecules with $U(4) \oplus U(4) \oplus U(4)[16]$. It has also been possible recently to study high order interactions such as rotation-vibration couplings.

\section{Conclusions}

Algebraic methods have been used in recent years in the study of molecular structure. When applied to this system, algebraic theory offers two main advantages: (i) The use of Lie algebras to describe the interaction (Morse, Pöschl-Teller, ...) allows one to extend the traditional harmonic analysis to anharmonic analysis. One can thus deal easily with highly excited states of molecules where anharmonicities play a crucial role (a subject of current experimental interest especially in connection with intramolecular relaxation and energy transfer.) (ii) The use of algebraic operators to couple the individual modes of a molecule allows one to construct symmetry adapted states in a simple way. One can thus deal with complex molecules where discrete symmetries play a crucial role.

The algebraic method can be used in molecules in two ways:

A) With rotation and vibrations treated separately. In this case the spectrum generating algebra is 


$$
\begin{aligned}
\mathcal{G} & =\mathcal{G}_{R O T} \oplus \mathcal{G}_{V I B} \\
\mathcal{G}_{V I B} & =\sum_{i} \oplus U_{i}(2) .
\end{aligned}
$$

B) With rotations and vibrations treated simultaneously. In this case the spectrum generating algebra is

$$
\mathcal{G}=\sum_{i} \oplus U_{i}(4)
$$

The latter case is more complete, but more difficult to treat than the former, since one has to deal with the Racah algebra of U(4).

In view of its simplicity, the method is particularly well suited for a study of complex systems such as macromoleculess, clusters, polymers, .... Work in this direction is in progress. An account of the algebraic theory of molecules is given in Ref.[17] and the mathematical formalism of Sects. 5 and 6 is reviewed in Ref.[18].

\section{Acknowledgements}

This work is supported in part by D.O.E. Grant DE-FG02-91ER40608. 


\section{References}

[1] Y. Dothan, M. Gell-Mann and, Y. Ne'eman, Phys.Lett. 17, 283 (1965); A.D. Barut and A. Böhm, Phys. Rev. 139, B1107 (1965).

[2] A. Arima and F. Iachello, Phys. Rev. Lett. 35, 1069 (1975).

[3] F. Iachello, Chem. Phys. Lett. 78, 581 (1981).

[4] F. Iachello, Nucl. Phys. A560, 23 (1993).

[5] B.G. Wybourne, 'Classical Groups for Physicists', J. Wiley and Sons, New York (1984), p. 286.

[6] P. Cordero and S. Hojman, Lett. Nuovo Cimento 4, 1123 (1970); R.D. Levine and C.E. Wulfman, Chem. Phys. Lett. 60, 372 (1979); M. Berrondo and A. Palma, J. Phys. A13, 773 (1980).

[7] Y. Alhassid, F. Gürsey and F. Iachello, Ann. Phys. (N.Y) 148, 346 (1983).

[8] F. Iachello and R.D. Levine, J. Chem. Phys. 77, 3046 (1982).

[9] F. Iachello and A. Arima, 'The Interacting Boson Model', Cambridge University Press, Cambridge (1987).

[10] F. Iachello and S. Oss, Phys. Rev. Lett. 66, 2976 (1991).

[11] F. Iachello and S. Oss, Chem. Phys. Lett. 187, 500 (1991).

[12] A. Frank and R. Lemus, Phys. Rev. Lett. 68 , 413 (1992).

[13] F. Iachello and S. Oss, J. Chem. Phys. 99, 7337 (1993).

[14] D. Bassi, L. Menegotti, S. Oss, M. Scotoni and F. Iachello, Chem. Phys. Lett. 207, 167 (1993).

[15] O. van Roosmalen, F. Iachello, R.D. Levine, and A.E.L. Dieperink, J. Chem. Phys. 79, 2515 (1983).

[16] F. Iachello, S. Oss and R. Lemus, J. Mol. Spectr. 149, 132 (1991).

[17] F. Iachello and R.D. Levine, 'Algebraic Theory of Molecules', Oxford University Press, Oxford (1994).

[18] A. Frank and P. van Isacker, 'Algebraic Methods in Molecular and Nuclear Structure Physics', J. Wiley and Sons, New York (1994). 